Results From 35 patients with Ng positive NAAT, we obtained 34 (97\%) Ng cultures from ESwab samples stored for 1 hour at RT. Storage for 24 hours at $4^{\circ} \mathrm{C}$ and RT resulted in $32(91 \%)$ cultures. Storage for 48 hours at $4^{\circ} \mathrm{C}$ yielded $25(71 \%)$, and at RT only 13 $(37 \%, p=0.007)$ cultures. Fourteen urine samples resulted in 13 (UR) respectively 14 (US) cultures after storage for 1 hour at RT. Storage for 24 hours at $4^{\circ} \mathrm{C}$ and RT resulted in 11 and 7 (UR), respectively 12 and 10 (US) cultures. Storage for 48 hours at $4^{\circ} \mathrm{C}$ and RT gave 3 and 1 (UR), respectively 5 and 2 (US) cultures.

Conclusion Delayed $\mathrm{Ng}$ cultivation from the ESwab system was successful after storage at $4^{\circ} \mathrm{C}$ for 24 hours in $91 \%$ and for 48 hours in $71 \%$ of cases. The ESwab system for NAAT diagnostics combined with delayed $\mathrm{Ng}$ cultivation of positive NAAT samples appears highly effective for future sustainable and essential gonococcal AMR surveillance. This approach is now being validated in routine practise.

\section{P5.078 FALSE-POSITIVE NEISSERIA GONORRHOEAE RESULTS IN URINE SAMPLES USING A HIGHLY SENSITIVE NAAT TESTS: THE SAMPLING SITE AS A SOURCE OF CONTAMINATION?}

doi:10.1136/sextrans-2013-051184.1122

\begin{abstract}
'2A P Van Dam, 'K Adams, 'I Linde, 'S Bruisten, 'A C G L Speksnijder, 3P Van Leeuwen, ${ }^{3,4} \mathrm{H} \mathrm{J}$ C De Vries. ' $P$ Public Health Laboratory, Cluster Infectious Diseases, Municipal Health Service, Amsterdam, The Netherlands; ${ }^{2}$ Onze Lieve Vrouwe Gasthuis, dept of Medical Microbiology, Amsterdam, The Netherlands; ${ }^{3} S T I$ outpatient clinic, Cluster Infectious Diseases, Municipal Health Service, Amsterdam, The Netherlands; ${ }^{4}$ Department of Dermatology, Academic Medical Center, Amsterdam, The Netherlands
\end{abstract}

Introduction False-positive results due to contamination of NAATs have been described. Apart from the laboratory, also the area where samples from patients are collected can be the source of the contamination.

Methods and results: In a 46 days period, 62 (7.3\%) of male patients visiting the STI outpatient clinic with a low risk for gonorrhoea showed a positive NAAT (AC 2, Hologic-GenProbe) for Neisseria gonorrhoeae (NG) in urine. This was only $0.8 \%$ in the previous 6 months. The prevalence of positive NAAT results for Chlamydia trachomatis (CT) remained unchanged. Culture was positive in only 2/24 NG-NAAT-positive patients whose cultures were available. The prevalence of NG among high-risk patients as determined by culturing, and the positive NG-NAAT results from vaginal, rectal and pharyngeal swabs from the STI clinic and from urines received from other practises remained unchanged.

All 5 environmental swabs from the male bathroom and all 4 swabs from transport trays were positive in NG-NAAT, but only 1 of these 9 was positive for CT. Swabs from trays from the laboratory, routinely cleaned with chlorine, were negative. An audit showed that some clients do not deliver their urine samples in a hygienic way and employees who transferred urine into Aptima tubes might have touched the seal of these tubes.

The pseudo-outbreak ended after daily cleaning of bathrooms and trays with chlorine and strictly following anti-contamination guidelines. Afterwards only $0.2 \%$ of low-risk male patients had a positive NG-NAAT in urine. Thirty-seven patients who had been treated for gonorrhoea were informed about the possible incorrect diagnosis.

Conclusion This pseudo-outbreak was most likely a consequence of external contamination of trays and test tubes with nucleic acids from the sampling site, in combination with inadequate handling of tubes during pipetting.

P5.079 LABORATORY DIAGNOSIS OF NEUROSYPHILIS IN PATIENTS CO-INFECTED WITH HUMAN IMMUNODEFICIENCY VIRUS (HIV) AND NEGATIVE-HIV PATIENTS IN MONTEVIDEOURUGUAY

doi:10.1136/sextrans-2013-051184.1123
R Balleste, N Rodriguez, N Garcia, P Lopez, C Buzzi. Clinical Hospital, Medicine Faculty, Republic University, Montevideo, Uruguay

Background Syphilis laboratory diagnosis, is made through the dosage of circulating antibodies in blood, but is not enough when neurological involvement is suspected.

A positive Venereal Disease Research Laboratory test (VDRL) result in cerebrospinal fluid (CSF) establishes the diagnosis of neurosyphilis, but it's negativity does not rule it out, therefore the need to use other immunological tests arises. The aim of our study was to evaluate the immunological tests performance used for neurosyphilis diagnosis and compare them between HIV infected and uninfected patients.

Methods We studied 37 patients (17 positive HIV and 20 negative HIV) from 2005 to 2012. We selected patients with positive Treponema Pallidum Hemagglutination Assay (TPHA) result in serum and CSF. Those patients underwent VDRL and, IgG and Albumin dosage in serum and CSF. TPHA-index and ITpA-index were calculated, intrathecal IgG production and indemnity of the blood brain barrier were evaluated through "Reiber's" diagram. Results:Neurosyphilis was diagnosed in 21 patients ( 6 positive HIV and 15 negative HIV): 18 reactive CSF VDRL, 13 TPHA in CSF > 1/320, 13 Index TPHA/Albumin $>70,24$ ITpA index $\geq 2$, in 3 patients neurosyphilis diagnosis was established only by high TPHA titers and high TPHA-index ( 2 positive HIV and 1 negative HIV).14 patients had Intrathecal IgG synthesis and 10 had blood-brain barrier disruption.

Conclusions CSF VDRL made neurosyphilis diagnosis in $86 \%$ of patients, with a high concordance between CSF VDRL and CSF TPHA titer $>1 / 320$ and TPHA-index $>70$; the immunological tests performance was similar in HIV positive and HIV negative patients. Neurosyphilis diagnosis was established with the CSF/serum indexes and TPHA titers in 14\% of the studied population. This highlights the importance of including the indexes in the routine diagnosis of neurosyphilis. Intrathecal IgG synthesis and disruption of the bloodbrain barrier predominated in patients with neurosyphilis.

\section{P5.080 COMPARISON OF AN IN-HOUSE POLYMERASE CHAIN REACTION AND DIRECT FLUORESCENT ANTIBODY ASSAY FOR DETECTION AND TYPING OF HERPES SIMPLEX VIRUS IN CLINICALLY SUSPECTED GENITAL HERPES}

doi:10.1136/sextrans-2013-051184.1124

P Bhalla, V Patwardhan. Maulana Azad Medical College, New Delhi, India

Background Molecular assays based on PCR have become an important tool for the detection of herpes simplex virus-1\&2 DNA in clinical specimens. Detection and typing of HSV can also be done by a monoclonal antibody based DFA. The present study was undertaken to standardise an in-house PCR for detection and typing of Herpes Simplex Virus (HSV) and compare it with Direct Fluorescent Antibody (DFA) test.

Methods 44 patients with genital herpes attending the STD clinic were studied. Specimens collected from genital lesions were placed in Viral transport medium (VTM) and stored at $-70^{\circ} \mathrm{C}$ till tested. DNA extraction was done using QiaAmp DNA mini kit (Qiagen, USA), PCR was carried out in GeneAmp PCR system 9700 (Applied BioSystems). Post PCR analysis of PCR product was done by electrophoresis using $2 \%$ agarose gel. DFA (BioRad) was also done for identification and typing of HSV-1\& 2 .

Results By DFA, 4 specimens were positive for HSV 1, 19 were positive for HSV 2 while 7 were positive for both. By PCR, 5 were HSV-1 positive, 18 were HSV-2 positive while 6 were positive for both HSV-1 \& 2. (kappa for HSV-1 = 0.879, HSV-2 = 0.63,) One HSV - 1 and 3 HSV-2 cases was positive by PCR but not by DFA. Four specimens that were positive by DFA but negative by PCR 
were retested in duplicate to rule out the presence of PCR inhibitors. Three of them were then found to have no inhibitors, while one had presence of PCR inhibitors.

Conclusions Our results show that although majority of cases studied were due to HSV-2, HSV-1 either alone or as a mixed infection with HSV-2 is not uncommon. PCR was found to be as sensitive as DFA for confirming the syndromic diagnosis, but some false negatives may occur due to presence of PCR infibitors.

\begin{tabular}{|c|c|c|c|c|}
\hline \multicolumn{5}{|l|}{ HSV1 DFA } \\
\hline HSV1 PCR & & Positive & Negative & Total \\
\hline Positive & 10 & 1 & 11 & \\
\hline Negative & 1 & 32 & 33 & \\
\hline Total & 11 & 33 & 44 & \\
\hline
\end{tabular}

\begin{tabular}{lllll} 
Abstract P5.080 & Table 2 & & \\
\hline HSV2 DFA & & & & \\
\hline HSV2 PCR & & Positive & Negative & Total \\
Positive & 21 & 3 & 24 & \\
Negative & 5 & 15 & 20 & \\
Total & 26 & 18 & 44 & \\
\hline
\end{tabular}

\section{P5.081 ANALYTICAL EVALUATION OF THE GENEXPERT ${ }^{\circledR}$ CT/ NG, THE FIRST GENETIC POINT OF CARE ASSAY FOR SIMULTANEOUS DETECTION OF NEISSERIA GONORRHOEAE AND CHLAMYDIA TRACHOMATIS}

doi:10.1136/sextrans-2013-051184.1125

1,2S N Tabrizi, ${ }^{3} \mathrm{M}$ Unemo, ${ }^{4} \mathrm{D}$ Golparian, ${ }^{5} \mathrm{~J}$ Twin, ${ }^{6} \mathrm{~A}$ E Limnios, ${ }^{6} \mathrm{M}$ Lahra, ${ }^{7} \mathrm{R}$ Guy, on behalf of TTANGO Investigators. 'Department of Microbiology and Infectious Diseases, The Royal Women's Hospital, Parkville, Australia; ${ }^{2}$ Department of Obstetrics and Gynaecology, University of Melbourne, The Royal Women's Hospital and Murdoch Childrens Research Institute, Parkville, Australia; ${ }^{3} \mathrm{WHO}$ Collaborating Centre for Gonorrhoea and other STIS, Department of Laboratory Medicine, Microbiology, Örebro University Hospital, Örebro, Sweden; ${ }^{43}$ ) WHO Collaborating Centre for Gonorrhoea and other STIS, Department of Laboratory Medicine, Microbiology, Örebro University Hospital, Örebro, Sweden; ${ }^{5}$ The Royal Women's Hospital, Parkville, Australia; $\left.{ }^{6} 4\right)$ WHO Collaborating Centre for STD, Microbiology Department, The Prince of UK Hospital, Randwick, Australia; ${ }^{7}$ Kirby Institute, University of NSW, Sydney, Australia

Introduction New assays for molecular detection of Neisseria gonorrhoeae and Chlamydia trachomatis need to be evaluated for potential false positive and false negative results due to cross reaction with other species, and potential mutations and genetic exchanges with other closely related organisms. Cepheid GeneXpert ${ }^{\circledR}$ CT/NG is the first FDA approved genetic point of care (POC) assay which simultaneously detects $C$. trachomatis, $N$. gonorrhoeae and controls for sample adequacy, in less than 90 minutes.

Method This study evaluated the GeneXpert ${ }^{\circledR}$ CT/NG assay with 372 characterised culture isolates; $111 \mathrm{~N}$. gonorrhoeae isolates (including 3 isolates with $N$. meningitidis porA sequence), 223 isolates of non-gonococcal Neisseria species, 13 isolates of other species closely related to Neisseria and 25 C. trachomatis strains of different serovars (including LGV and nvCT strains).

Results All C. trachomatis and $N$. gonorrhoeae isolates were detected. A detection sensitivity of 10 genome copies per reaction was obtained with all C. trachomatis serovars as well as a representative $N$. gonorrhoeae control strain. Among the 223 non-gonococcal isolates, 4/11 N. mucosa and 2/42 N. subflava isolates were positive in one of the two $N$. gonorrhoeae targets (NG4), however the assay flagged all 6 as negative due to requirements of both NG targets being positive for the assay to display an $N$. gonorrhoeae positive result

Conclusion GeneXpert ${ }^{\circledR}$ CT/NG assay was analytically highly sensitive and specific for detection of $C$. trachomatis and $N$. gonorrhoeae, showing great promise as a POC assay. Detection of two NG targets is highly advantageous for avoiding false positive $N$. gonorrhoeae results.

\section{P5.082 WHAT ARE THE COSTS AND BENEFITS OF IMPLEMENTING POINT OF CARE TESTS FOR CHLAMYDIA TRACHOMATIS AND NEISSERIA GONORRHOEAE IN GENITOURINARY MEDICINE CLINICS?}

doi:10.1136/sextrans-2013-051184.1126

${ }^{1} \mathbf{K}$ M Turner, ${ }^{2} \mathrm{~J}$ Round, ${ }^{3} \mathrm{~A}$ Deol, 'P Horner, ' $\mathrm{J}$ Macleod, ${ }^{4} \mathrm{~S}$ Goldenberg, ${ }^{3} \mathrm{E}$ Adams. ${ }^{1}$ University of Bristol, Bristol, UK; ${ }^{2}$ University College London, London, UK; ${ }^{3}$ Aquarius Population Health, London, UK; 'Guy's \& St. Thomas' NHS Foundation Trust and King's College London, London, UK

Background To estimate the costs and benefits of new patient pathways in genitourinary medicine (GUM) clinics that incorporate a point of care (POC) nucleic acid amplification test (NAAT) for chlamydia and gonorrhoea (CT/NG), compared to standard off-site laboratory testing.

Method We modelled 20,000 GUM clinic attendees, based on GUMCAD reported diagnoses for men and women in England (2011). A Markov model with Monte Carlo simulation in Excel was developed. We compared existing standard pathways of testing and treatment using a CT/NG test with a new POC NAAT. Scenario and sensitivity analyses were conducted to evaluate the robustness of the model findings. The primary outcome was incremental cost effectiveness ratio (ICER, $£$ per QALY) of testing and treatment in GUM clinics. Secondary outcomes included the number of overtreatments for CT/NG, complications and transmissions averted, and change in time from test to treatment.

Results The total cost of using the CT/NG POC NAAT in 20,000 patients was $£ 1.73$ million and $£ 1.92$ million for standard care. The new POC NAAT pathway dominated (less expensive and increased QALYs, ICER of $£ 4,397 /$ OALY saved). As many as 541 unnecessary treatments could be prevented using POC NAAT. The shorter time to treatment for patients receiving same-day diagnosis and treatment may also prevent a small number of complications (3.4 cases PID) and onward transmissions (31.9 infections).

Discussion Replacing standard laboratory tests for CT/NG with a POC NAAT seems to be cost saving or at least cost neutral, and patients would benefit from more accurate diagnosis and less unnecessary treatment. POC NAATs would effectively eliminate the need for presumptive treatment.

\section{P5.083 WHAT QUALITIES DO PROVIDERS IDENTIFY AS BEST FOR POINT OF CARE STI TESTS AND DO OPINIONS DIFFER BY PRACTISE, REGION AND COUNTRY?}

doi:10.1136/sextrans-2013-051184.1127

${ }^{1}$ A M Rompalo, ${ }^{1} Y$ Hsieh, ${ }^{2} \mathrm{R}$ Guy, ${ }^{2} \mathrm{~L}$ Natoli, ${ }^{3} \mathrm{C}$ Fairley, ${ }^{4} \mathrm{~S}$ Gupta, ${ }^{5} \mathrm{~K}$ Radcliffe, ${ }^{1} \mathrm{M} \mathrm{T}$ Hogan, 'M J Goheen, 'C Gaydos. 'Johns Hopkins School of Medicine, Baltimore, MD, United States; ${ }^{2}$ The Kirby Institute University of New South UK, Coogee, Australia; ${ }^{3}$ University of Melbourne, Carlton, Australia; ${ }^{4}$ All India Institute of Medical Sciences, New Dehli, India; ${ }^{5}$ University Hospitals Birmingham, Birmingham, UK

Background U.S. clinicians identified high sensitivity and low cost as the most desirable characteristics for a Sexually Transmitted Infection (STI) Point Of Care Test (POCT); indicated performance time as major barrier; and chose Chlamydia trachomatis as the first choice for POCT development. We determined if POCT priorities, preferred qualities and barriers were similar for practitioners globally. 